\title{
Bioinspired Simultaneous Changes in Fluorescence Color, Brightness and Shape of Hydrogels Enabled by AlEgens
}

\author{
Zhao $\mathrm{Li}^{1,2}$, Xiaofan $\mathrm{Ji}^{1,2}$, Junyi Gong ${ }^{1,2}$, Yubing $\mathrm{Hu}^{1,2}$, Wenjie $\mathrm{Wu}^{1,2}$, Xinnan Wang ${ }^{1,2}$, Hui-Qing \\ Peng $^{1,2}$, Ryan T. K. Kwok ${ }^{1,2}$, Jacky W. Y. Lam ${ }^{1,2, *}$ and Ben Zhong Tang ${ }^{1,2,3, *}$
}

${ }^{1}$ Department of Chemistry, Hong Kong Branch of Chinese National Engineering Research Center for Tissue Restoration and Reconstruction and Institute for Advanced Study, The Hong Kong University of Science and Technology, Clear Water Bay, Kowloon, Hong Kong, China.

${ }^{2}$ HKUST-Shenzhen Research Institute, No. 9 Yuexing 1st RD, South Area, Hi-tech Park, Nanshan, Shenzhen 518055, China.

${ }^{3}$ Center for Aggregation-Induced Emission, SCUT-HKUST Joint Research Institutes, State Key Laboratory of Luminescent Materials and Devices, South China University of Technology, Guangzhou, 510640, China.

KEYWORDS: simultaneous, changes, fluorescence color, fluorescence brightness, complex shape, stimuli-responsive, aggregation-induced emission luminogens (AIEgens), bilayer hydrogel actuators.

\begin{abstract}
Development of stimuli-responsive materials with complex practical functions is significant for achieving bioinspired artificial intelligence. It is challenging to fabricate stimuli-responsive hydrogels showing simultaneous changes in fluorescence color, brightness and shape in response to one stimulus. Herein a bilayer hydrogel strategy was designed by utilizing an aggregation-induced emission luminogen (AIEgen) tetra-(4-pyridylphenyl)ethylene (TPE-4Py) to fabricate hydrogels with the above capabilities. Bilayer hydrogel actuators with ionomer of poly(acrylamide-r-sodium 4styrenesulfonate) (PAS) as matrix of both active and passive layers and TPE-4Py as the core function element in the active layer were prepared. At acidic $\mathrm{pH}$, the protonation of TPE-4Py led to fluorescence color and brightness changes of the actuators and the electrostatic interactions between the protonated TPE-4Py and benzenesulfonate groups of PAS chains in the active layer caused the actuators to deform. The proposed TPE-4Py/PAS-based bilayer hydrogel actuators with such responsiveness to stimulus provide pregnant insights in the design of intelligent systems and are highly attractive material candidates in fields of $3 \mathrm{D} / 4 \mathrm{D}$ printing, soft robots and smart wearable devices.
\end{abstract}

\section{INTRODUCTION}

In nature, numerous biological systems are intelligent. ${ }^{1,2}$ Many species are able to simultaneously change their colors and shapes in response to stimulus. For an example, chameleons can rapidly alter their skin color when they move into a new place to blend into its different surrounding environment. ${ }^{3}$ Mimic octopuses, on the other hand, are able to change both their color and shape in order to camouflage or hunt. ${ }^{4,5}$ Frilled-necked lizards spread out their frills and the frills simultaneously turn to bright yellow, orange or red when they are frightened or during courtship. ${ }^{6,7}$ Besides animals, some plants show also the ability to simultaneously change their colors and shapes. Brunfelsia acuminata also known as "YesterdayToday-Tomorrow" of which flowers open purple, fade to lavender and finally to white during blooming process is a typical example. ${ }^{8,9}$ Quisqualis indica, on the other hand, has white and horizontally oriented flowers that open at dusk and change to pink and later to red on the following day. The flowers become pendulous by simultaneously changing their orientation during their florescence. ${ }^{10,11}$ Tibouchina pulchra flora color changes from white in the first day to pink in the following days of anthesis. ${ }^{12}$ Such a process of simultaneous color and shape changes of the flowers is interpreted as a warning mechanism for pollinators to avoid old flowers. ${ }^{13}$

Scientific researchers are pursuing to fabricate freestanding intelligent artificial materials. One promising approach is to use stimuli-responsive materials. ${ }^{14,15} \mathrm{Be}-$ cause of this, they have been capturing much interest. ${ }^{16-18}$ Among them, smart hydrogel ${ }^{19-22}$ is an important member and can change its volume or other properties dramatically under stimuli such as temperature, ${ }^{23-25} \mathrm{pH}^{26,27}$ irradiation, ${ }^{28,29}$ electric field ${ }^{30}$ magnetic field, ${ }^{31}$ ionic strength, ${ }^{32}$ a specific chemical ${ }^{33,34}$ and so on. Inspired by the biological behaviors, a number of attempts have been reported to construct stimuli-responsive hydrogels with capacities of undergoing shape deformation ${ }^{21,35-43}$ in the presence of stimulus utilizing the bilayer technique ${ }^{44}$ or color change 
including fluorescence change. ${ }^{45-48}$ However, most of the hydrogels show either of shape change or color change. Although there are very few reports on deformable hydrogels showing stimulus-induced fluorescence color change, the shape change and the fluorescence change are independent. The two changes are based on different elements in the hydrogel or are triggered by different stimuli. It is thus challenging to realize response hydrogels with simultaneous changes of fluorescence color, brightness and shape based on one stimulus.

It is critical to select a suitable fluorophore as the sole source of the changes to realize the above goal. Aggregation-induced emission luminogens (AIEgens) ${ }^{49}$ are nonemissive as isolated species in good solvents but are highly emissive in poor solvents as aggregates. ${ }^{50}$ Such an aggregation-induced emission (AIE) phenomenon is believed to be caused by the restriction of intramolecular motion (RIM) in the aggregated state..$^{51}$ The strong emission of AIEgens in the aggregated, solid or restricted states makes them suitable to be applied in semi-solid hydrogel systems..$^{52,53}$ As a burgeoning scientific research field, AIE has attracted intense interest and has been applied in numerous areas. ${ }^{54-57}$ The fast and wide development of AIEgens is due to their superiorities of comprehensible mechanism, accessible designability, easy functionalization, large absorptivity, high brightness in the restricted state and low background noise. Until now, a large variety of stimuli-responsive AIEgens have been developed and applied, ${ }^{58-63}$ and many of them experience fluorescence color or brightness changes when they are ionized $^{64-69}$ and are potential building blocks for the construction of supramolecular materials. ${ }^{70,71}$ Therefore, AIEgens are ideal candidates in the design and fabrication of stimuli-responsive hydrogel systems fluorescence color, brightness and shape changes in response to stimulus.

In this study, we reported the design and construction of bioinspired hydrogels with abilities of simultaneous fluorescence color and intensity changes as well as complex shape deformation by utilizing AIEgens and the bilayer hydrogel technique. ${ }^{44}$ The $\mathrm{pH}$-responsive AIEgen called tetra-(4-pyridylphenyl)ethylene (TPE-4Py) was used to impart the formed bilayer hydrogel actuators with the above changes and an ionomer namely poly(acrylamide- $r$-sodium 4-styrenesulfonate (PAS) was used as the matrix of the actuators. When the actuators were immersed in acidic aqueous solution, they could change their fluorescence color, brightness and actuator shape simultaneously. The ability for shape change was achieved by asymmetrical distribution of TPE-4Py aggregates in the two layers of the actuators. At low $\mathrm{pH}$, the protonation of TPE-4Py embedded in the active layer of the actuators caused altered fluorescence color and intensity. Meanwhile, electrostatic interactions between the protonated TPE-4Py and the benzenesulfonate groups of PAS chains led to the shape deformation of the actuators. The resulting TPE-4Py/PAS-based bilayer hydrogel actuators with simultaneous stimulus-induced fluorescence color, brightness and complex shape changes provide new insights in the design of advanced bioinspired intelligent systems with complex practical functions and may have potential applications in fields of $3 \mathrm{D} / 4 \mathrm{D}$ printing, soft robots and smart drug delivery systems.

\section{RESULTS AND DISCUSSION}

Design of Bilayer Hydrogel Actuators with Simultaneous Fluorescence Color, Brightness and Complex Shape Changing Property. It is a key that the fluorescence color changing function, the fluorescence brightness changing function and the complex shape deformation function are built based on one element in the hydrogel actuator to realize its ability of simultaneous fluorescence color, brightness and complex shape changing. AIEgen is a good candidate of the element. TPE-4Py, a classical AIEgen with $\mathrm{pH}-$ responsiveness was selected..$^{72-74}$ It can form aggregates in water with neutral $\mathrm{pH}$ to form a suspension due to its hydrophobicity. The aggregates emit strong sapphire fluorescent light based on the effect of the restriction of intramoelcular rotation (RIR) ${ }^{75,76}$ When the $\mathrm{pH}$ value of the suspension is decreased, TPE-4Py is able to protonates to form the tetra-protonated TPE- $4 \mathrm{Py}\left(4 \mathrm{H}-\mathrm{TPE}-4 \mathrm{Py}^{4+}\right)$ and the fluorescence color red shifts due to the enhanced intramolecular charge transfer (ICT) process. ${ }^{77,78}$ In addition, the protonation of TPE-4Py increases the solubility of it largely, which reduces the fluorescence brightness obviously due to the enhancement of intramoelcular rotation (EIR) effect. Taking advantage of the protonation process of TPE-4Py during the $\mathrm{pH}$ decreasing process, an ionomer PAS that carries negatively charged groups was used as the matrix of the actuator. The PAS hydrogel was fabricated through in situ random copolymerization of monomers acrylamide (AAm) and sodium 4-styrenesulfonate (NaSS) and a cross-linker $N, N^{\prime}$-methylenebisacrylamide (BIS). TPE-4Py/PAS-based bilayer hydrogel actuators with TPE-4Py as the functional element and PAS hydrogel as the matrix were designed, in which a PAS hydrogel layer acted as the passive layer and a TPE-4Py aggregates encapsulated PAS hydrogel layer acted as the active layer (Figure 1a). In the neutral medium, the TPE-4Py aggregates were physically encapsulated in the PAS hydrogel in the active layer and didn't have interactions with network chains of the hydrogel. When the bent bilayer hydrogel actuator was immersed in an acidic aqueous solution, TPE-4Py in the aggregates protonated and dissolved gradually, and electrostatic interactions were occurred between protonated pyridyl groups in the formed $4 \mathrm{H}$ TPE-4Py ${ }^{4+}$ and benzenesulfonate groups on hydrogel network chains in the active layer (Figure $1 \mathrm{~b}$ ). The $4 \mathrm{H}$ TPE-4Py ${ }^{4+}$ acted as the additional physical cross-linking point and caused the active layer to shrink, and the hydrogel actuator gradually unbent. ${ }^{79}$ Meanwhile, the fluorescence color of the hydrogel actuator red shifted gradually due to the protonation of TPE-4Py. Because the dissolved $4 \mathrm{H}-\mathrm{TPE}-4 \mathrm{Py}^{4+}$ formed electrostatic interactions with network ionomer chains, the intramolecular rotation of $4 \mathrm{H}-\mathrm{TPE}-4 \mathrm{Py}^{4+}$ were restricted. Thus, the brightness of the red shifted fluorescent light enhanced and visualized. 
(a)
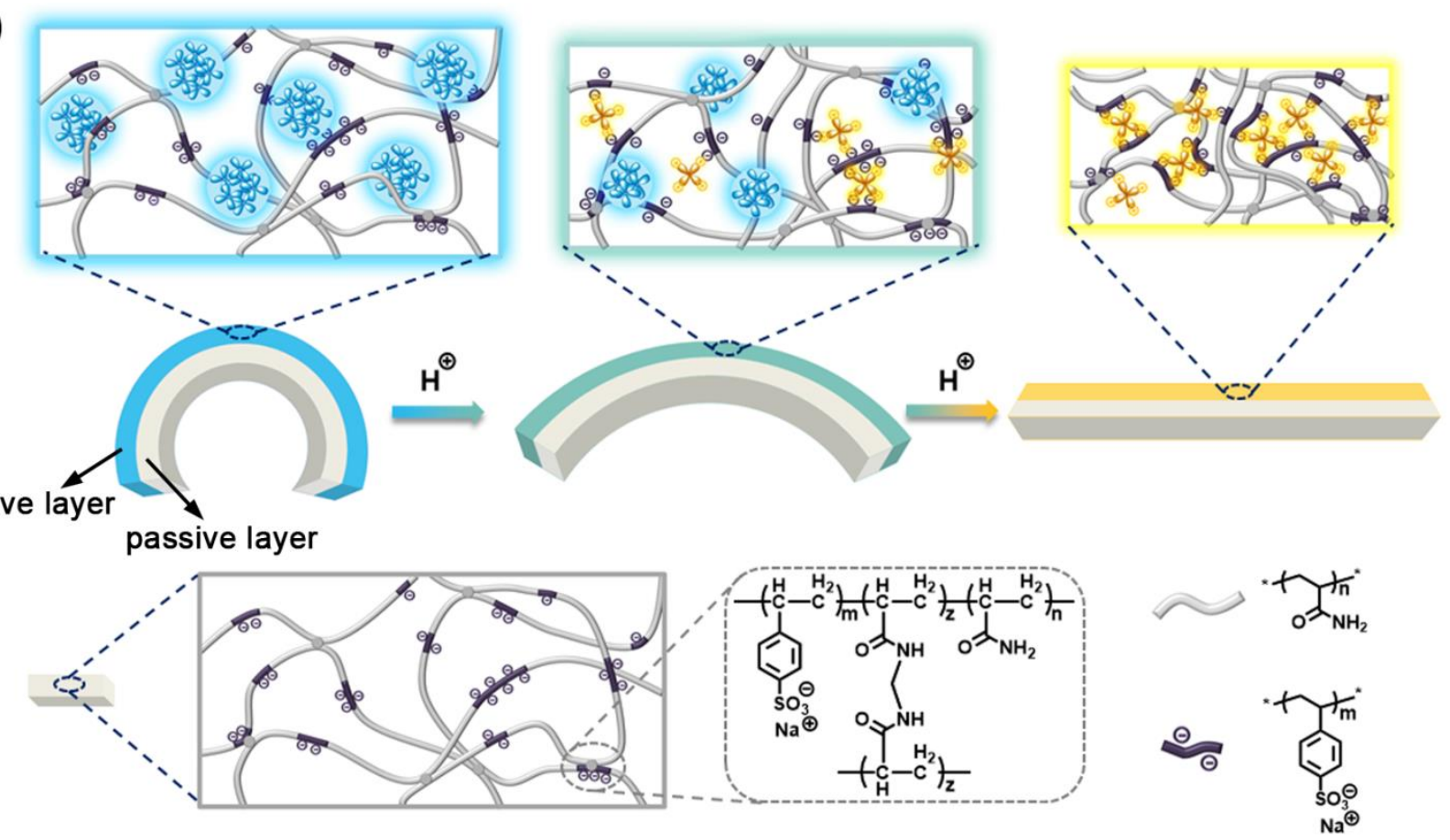

TPE-4Py aggregate
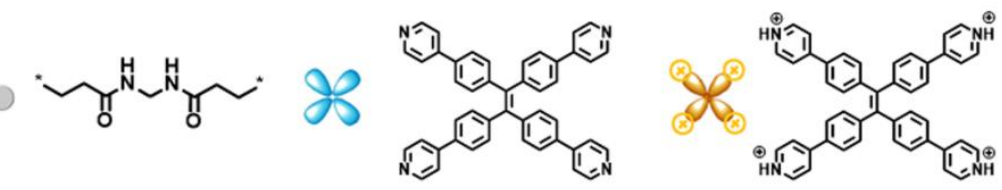

(b)
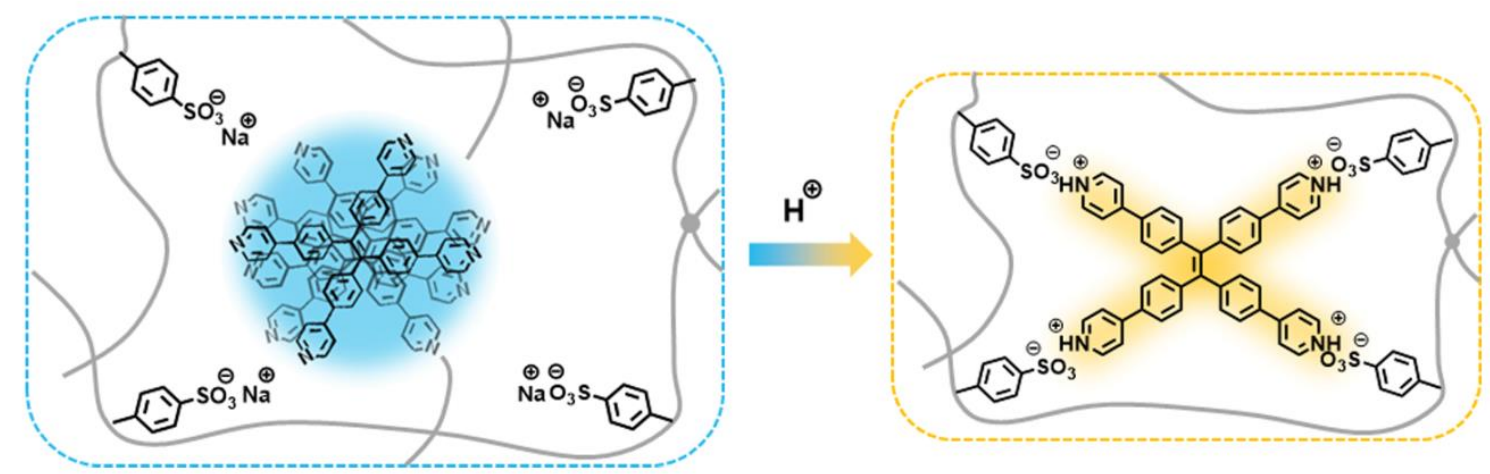

Figure 1. (a) Schematic illustration of simultaneous changes in fluorescent color and brightness and $3 \mathrm{D}$ shape of a TPE-4Py/PASbased bilayer hydrogel actuator and the mechanism involved. (b) When the medium of the hydrogel actuator becomes acidic, the TPE-4Py in an aggregate is protonated and dissolves, and electrostatically interacts with benzenesulfonate groups on network chains in the active layer.

The pH-Responsiveness of TPE-4Py. As the core function element in bilayer hydrogel actuators in the present work, TPE-4Py was first investigated. A series of TPE${ }_{4} \mathrm{Py}$ aqueous suspensions with different $\mathrm{pH}$ values from 2.09 to 10.13 were prepared using $\mathrm{pH}$ buffers. Figure $2 \mathrm{a}$ shows their photographs under 365-nm UV illumination. Their PL spectra were measured (Figure $2 \mathrm{~b}$ ) and the $\mathrm{pH}$ dependences of the wavelength at the maximum PL intensity $\left(\lambda_{\max }\right)$ and the maximum PL intensity $\left(I_{\max } / I_{0}\right)$ were depicted in Figure $2 \mathrm{c}$. It can be seen from Figure $2 \mathrm{c}$ that $\lambda_{\max }$ has little change and $I_{\max } / I_{\mathrm{o}}$ increases slightly with $\mathrm{pH}$ increasing from 6.98 to 10.13 . When the $\mathrm{pH}$ value decreas- es from 6.98 to $2.09, \lambda_{\max }$ first decreases slightly, then increases, followed by a sharp decline and a sharp large increment, and reaches the equilibrium of about $536 \mathrm{~nm}$ with $\mathrm{pH}$ value below 2.96. Meanwhile, with the $\mathrm{pH}$ value decreasing from 6.98 to $2.09, I_{\max } / I_{\mathrm{o}}$ first decreases slightly, then increases, followed by a sharp large decrease, and reaches the equilibrium with $\mathrm{pH}$ value below 3.58. In neutral medium, TPE-4Py forms aggregates in amorphous state (Figure $\mathrm{S}_{7}$, $\mathrm{S} 8$ and $\mathrm{S}_{9}$ ) in the aqueous buffer solution. The restriction of intramolecular rotation of TPE-4Py leads to the strong sapphire emission. When the $\mathrm{pH}$ value gradually decreases into the faintly acidic range, the solu- 
(a)

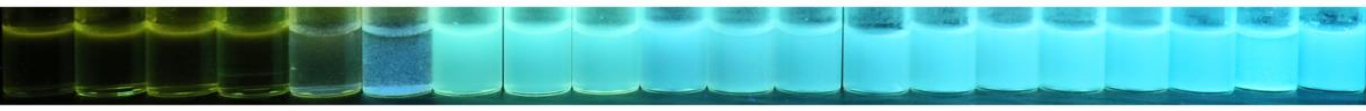

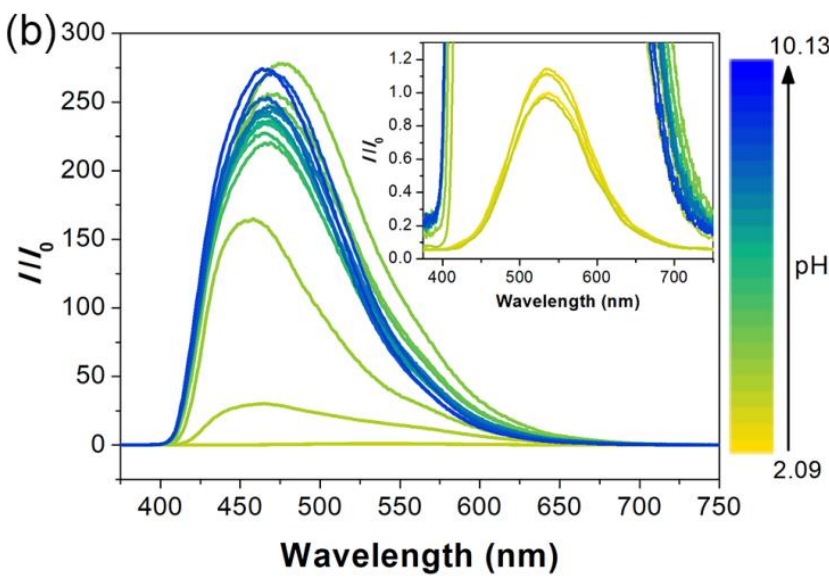

(d)

HOMO

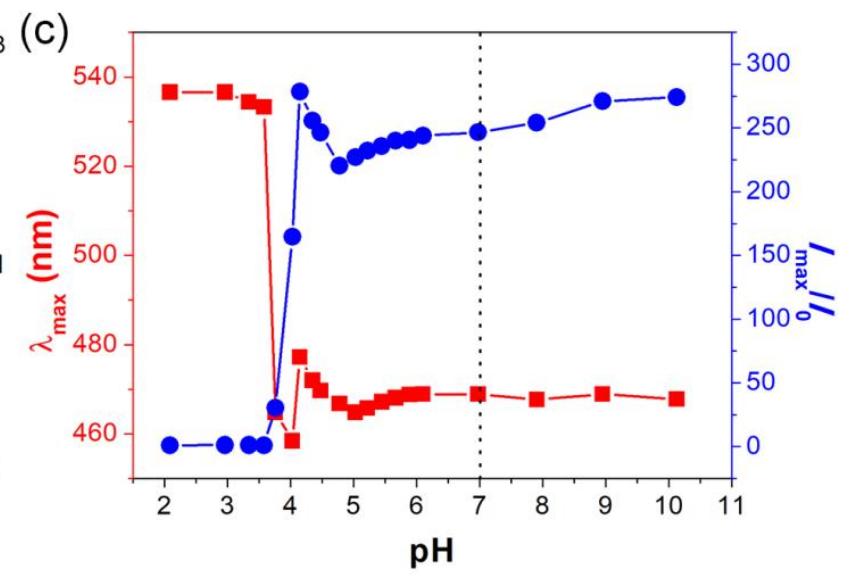

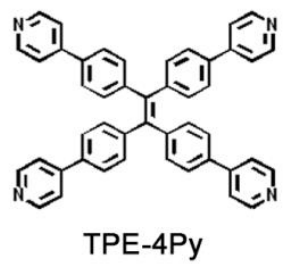

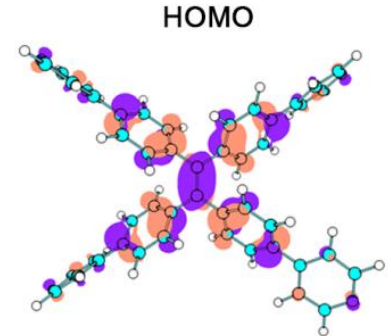

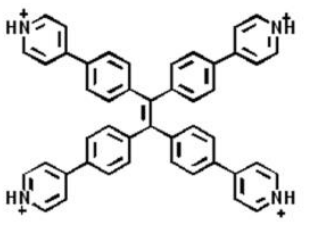

4H-TPE-4Py ${ }^{4+}$

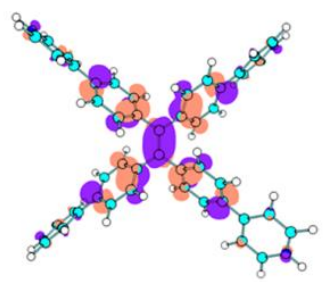

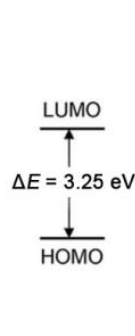

LUMO
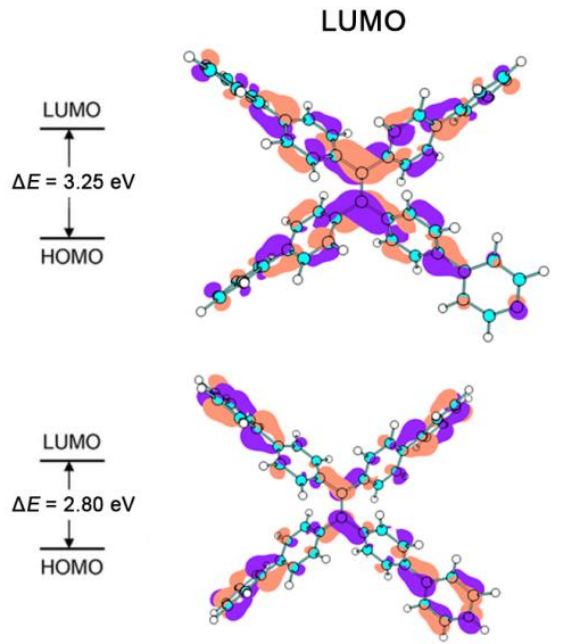

Figure 2. (a) Photographs of TPE-4Py aqueous suspensions $\left(c=10^{-4} \mathrm{M}\right.$ ) with different $\mathrm{pH}$ values. (pH values of the suspensions from left to right are 2.09, 2.96, 3.34, 3.58, 3.76, 4.03, 4.15, 4.35, 4.48, 4.78, 5.03, 5.22, 5.45, 5.67, 5.89, 6.10, 6.98, 7.91, 8.95 and 10.13 respectively.) (The photographs were taken under 365-nm UV illumination.) (b) PL spectra of the suspensions in (a). Insets are amplified PL spectra with Y axis being magnified with 230 times. ( $I$ is the PL intensity of the suspensions with different $\mathrm{pH}$ values and $I_{\mathrm{o}}$ is the maximum PL intensity of the suspension with $\mathrm{pH}$ of 2.09.) $\left(\lambda_{\mathrm{ex}}=365 \mathrm{~nm}\right)$ (c) Wavelength at the maximum PL intensity $\left(\lambda_{\max }\right)$ and the maximum PL intensity $\left(I_{\max } / I_{\mathrm{o}}\right)$ of the suspensions as a function of $\mathrm{pH}$. ( $I_{\max }$ is the maximum PL intensity of the suspensions with different $\mathrm{pH}$ values.) (d) Molecular structures and molecular orbital amplitude plots of HOMO and LUMO energy levels of TPE-4Py and ${ }_{4} \mathrm{H}-\mathrm{TPE}-4 \mathrm{Py}^{4+}$.

bility of TPE-4Py increases gradually and the fluorescent intensity of the suspension decreases slightly due to the EIR effect. When the $\mathrm{pH}$ value continues to decrease below 5 (from 4.78 to 4.15 ), TPE-4Py gradually protonates. The emerging obvious intramolecular charge transfter (ICT) process causes the fluorescent emission to red shifts. Meanwhile, because the solubility of TPE-4Py further increases, nano-crystals of it form which was demonstrated by scanning electron microscope (SEM) and polarizing optical microscope (POM) (Figure S8 and S9). Crystallization-enhanced emission (CEE) effect ${ }^{59}$ leads to the increment of the fluorescent intensity of the suspension. With the $\mathrm{pH}$ value continuing to decrease to $3 \cdot 76$, further in- crease of the solubility leads to the formation of TPE-4Py crystal (Figure S7, S8 and S9) which rendering the blue shifted fluorescent emission. ${ }^{80,81}$ The CEE effect is supposed to lead to fluorescent intensity increase. However, a mass of TPE-4Py molecules protonate and dissolve in this $\mathrm{pH}$ medium. The EIR effect causes their emission pretty weak. Thus the suspension shows reduced intensity and blue shifted emission. When the $\mathrm{pH}$ value further decreases below 3.7, all TPE-4Py molecules protonate to ${ }_{4} \mathrm{H}$ TPE-4 $\mathrm{Py}^{4+}$ with yellow emission. The dissolution of the ${ }_{4} \mathrm{H}$-TPE-4 $\mathrm{Py}^{4+}$ activates its intramolecular rotation, leading to a very weak fluorescent intensity. 
DFT calculation was used to simulate the molecular orbital amplitudes of HOMO and LUMO energy levels of TPE-4Py and ${ }_{4} \mathrm{H}-\mathrm{TPE}-4 \mathrm{Py}^{4+}$ (Figure $2 \mathrm{~d}$ ). Based on the calculated energy gaps $(\Delta \mathrm{E})$ between HOMO and LUMO energy levels, UV-Vis absorption spectra of TPE-4Py and ${ }_{4} \mathrm{H}-\mathrm{TPE}-4 \mathrm{Py}^{4+}$ were predicted in Figure Sioa. UV-Vis spectra of $\mathrm{TPE}-4 \mathrm{Py}$ in $\mathrm{CHCl}_{3}$ and $\mathrm{TFA} / \mathrm{CHCl}_{3}$ mixture (1/9, $\mathrm{V} / \mathrm{V}$ ) were measured (Figure Siob). The spectrum of TPE${ }_{4} \mathrm{Py}$ in the $\mathrm{TFA} / \mathrm{CHCl}_{3}$ mixed solvent is consistent with that of predicted ${ }_{4} \mathrm{H}-\mathrm{TPE}-4 \mathrm{Py}^{4+}$. In addition, ${ }^{1} \mathrm{H} \mathrm{NMR}$ spectrum in Figure $3 \mathrm{e}$ (II) of the $\mathrm{D}_{2} \mathrm{O}$ solution of TPE-4Py with $\mathrm{pH}$ value of 2.0 also demonstrates TPE-4Py transforms into ${ }_{4} \mathrm{H}-\mathrm{TPE}-4 \mathrm{Py}^{4+}$ at this $\mathrm{pH}$ value. Thus, in the TFA/ $\mathrm{CHCl}_{3}$ mixed solvent, TPE-4Py protonates into ${ }_{4} \mathrm{H}$ TPE-4 $\mathrm{Py}^{4+}$. UV-Vis absorption spectra of TPE-4Py aqueous suspensions with $\mathrm{pH}$ values from 2.09 to 4.03 were also measured (Figure S10c) and they are all consistent with the spectrum of ${ }_{4} \mathrm{H}-\mathrm{TPE}-4 \mathrm{Py}^{4+}$. Therefore, TPE-4Py protonates to form $4 \mathrm{H}-\mathrm{TPE}-4 \mathrm{Py}^{4+}$ with $\mathrm{pH}$ value below 4 .

The pH-Responsive Fluorescence Color, Brightness and Volume Changing Properties of TPE${ }_{4} \mathrm{Py} / \mathrm{PAS}$-Based Monolayer Hydrogels. After the $\mathrm{pH}-$ responsiveness of TPE-4Py in its aqueous suspension was made clear, monolayer hydrogels based on the TPE-4Py aggregate and the PAS hydrogel were constructed. Mi to M5 monolayer hydrogel film (thickness: $1 \mathrm{~mm}$ ) samples with increased TPE-4Py concentration were prepared according to recipes in Table S1. PAS hydrogel Mo without TPE-4Py was also prepared as the blank sample for the comparison. The hydrogel films were swollen in DI water to the equilibrium before further experiments.

Rectangular hydrogel sheets of Mo to M5 with the size of about $10 \mathrm{~mm} \times 10 \mathrm{~mm}$ were immersed in aqueous solutions with $\mathrm{pH}$ values from 7.30 to 1.85 for $17 \mathrm{~h}$. Photographs of the samples under day-light and 365-nm UV illumination were shown in Figure 3 a and Figure S11. Fluorescent emission spectra (Figure S12) of the samples were measured and $\mathrm{pH}$ dependences of the $\lambda_{\max }$ and the maximum PL intensity $\left(I_{\max }\right)$ were depicted in Figure $3 \mathrm{~b}$ and c. From the photographs and the figures, it can be seen that with the decrease of the $\mathrm{pH}$ value, the fluorescent emissions of M1 to M5 red shift and the fluorescent intensities of them decrease gradually. Because the blank sample Mo dose not have fluorescent emission (Figure 3c), the origin of the fluorescent emission of the monolayer hydrogels is TPE-4Py. When the $\mathrm{pH}$ value decreases, TPE-4Py in the aggregate in the monolayer hydrogel sample gradually protonates, which leads to the red-shift of the hydrogel sample. Meanwhile, the increase of the solubility of protonated TPE-4Py causes the decrease of its fluorescent intensity due to the EIR effect though the electrostatic interaction between formed pyridyl groups and benzenesulfonate groups on PAS chains restricts the intramolecular rotation of the protonated TPE-4Py to some extent. It can also be seen from the Figure $3 \mathrm{C}$ that with the increase of the TPE-4Py concentration from Mi to $\mathbf{M}_{5}$, the fluorescent intensity of the hydrogel sample at a specific $\mathrm{pH}$ value increases. This phenomenon can be easily understood that with the increase of the TPE-4Py concentration, the amount of fluorophores increases.

It can also be seen from Figure 3 a that with the decrease of the $\mathrm{pH}$ value, volumes of the monolayer hydrogel samples of Mi to M5 decrease. Masses of Mo to M5 before $\left(m_{\mathrm{o}}\right)$ and after $\left(m_{\mathrm{e}}\right)$ immersing were measured and the shrinkage ratios $(\eta)$ of them were calculated by the following equation:

$$
\eta=\frac{m_{0}-m_{e}}{m_{0}} \times 100
$$

$\mathrm{pH}$ dependences of $\eta$ of Mo to $\mathbf{M}_{\mathbf{5}}$ were depicted in Figure $3 \mathrm{~d}$. With the decrease of the $\mathrm{pH}$ value, hydrogels Mo to M5 shrink. $\eta$ of Mo slightly increases with $\mathrm{pH}$ value decreasing from 7.30 to 3.0o. When the $\mathrm{pH}$ value further decreases, $\eta$ of Mo increases largely up to $59.72 \%$. This may be caused by the ion-shield effect at low $\mathrm{pH}$ medium. With the introduction of TPE-4Py aggregates, $\eta$ of the hydrogel sheets increases compared with that of Mo in acidic medium. This is caused by the emerged electrostatic interactions between protonated TPE-4Py and benzenesulfonate groups on the hydrogel network chains. In acidic medium, protonated TPE-4Py acts as additional physical cross-linking point. The increase of the crosslinking density causes the hydrogel to shrink. With the increase of the TPE-4Py concentration, $\eta$ increases from M1 to $\mathrm{M}_{5}$ at a specific $\mathrm{pH}$ value. This can be easily understood that the higher the TPE-4Py concentration is, the more amount of physical cross-linking points generate in acidic medium.

NMR spectroscopy was used to demonstrate the existence of the electrostatic interaction between protonated TPE-4Py and benzenesulfonate groups on the hydrogel network chains. A linear random copolymer PAAm-r-PSS was synthesized (see Supporting Information) as the modal of the hydrogel network chain. A $\mathrm{D}_{2} \mathrm{O}$ solution of PAAm- $r$-PSS, a $\mathrm{D}_{2} \mathrm{O}$ solution of TPE-4Py and a $\mathrm{D}_{2} \mathrm{O}$ solution containing both PAAm-r-PSS and TPE-4Py with the $\mathrm{pH}$ value of 2.0 were prepared. $\mathrm{A}_{2} \mathrm{O}$ solution containing both PAAm-r-PSS and TPE-4Py with the $\mathrm{pH}$ value of 7.0 was also prepared. ${ }^{1} \mathrm{H}$ NMR spectra of the solutions were taken and shown in Figure ze. From the ${ }^{1} \mathrm{H}$ NMR spectrum of the PAAm-r-PSS solution (Figure ze (I)), it can be seen that signals of protons $\mathrm{H}_{\mathrm{a}}$ and $\mathrm{H}_{\mathrm{b}}$ on the benzenesulfonate group on PAAm-r-PSS are located at 7.72 and 7.33 ppm. The shapes and chemical shifts of proton signals on PAAm- $r$-PSS in Figure ze (I) are identical with that on PAAm- $r$-PSS in $\mathrm{D}_{2} \mathrm{O}$ with the $\mathrm{pH}$ value of 7.0 in Figure $\mathrm{S}_{3}$. There are four doublet peaks at 8.73, 8.26, 7.8o and 7.48 in the ${ }^{1} \mathrm{H}$ NMR spectrum of TPE- 4 Py solution in Figure $3 \mathrm{e}$ (II), which are assigned to protons $\mathrm{H}_{1}, \mathrm{H}_{2}, \mathrm{H}_{3}$ and $\mathrm{H}_{4}$ respectively on $4 \mathrm{H}-\mathrm{TPE}-4 \mathrm{Py}^{4+}$. When PAAm-r-PSS and TPE${ }_{4} \mathrm{Py}$ were mixed in $\mathrm{D}_{2} \mathrm{O}$ in neutral medium, the ${ }^{1} \mathrm{H}$ NMR spectrum of the mixture in Figure ze (III) only contains signals of PAAm-r-PSS protons. TPE-4Py in the aggregation state dose not have any proton signals. The spectrum in Figure ze (III) is identical with the spectrum in Figure ze (I). This indicates that PAAm-r-PSS and TPE-4Py aggregate do not have interactions. When the $\mathrm{pH}$ 
M5 (under day-light)

\section{L- M5 (under 365-nm UV) \\ $-\nabla$ - M4 (under 365-nm UV) \\ - 1 M3 (under 365-nm UV) \\ - - M2 (under 365-nm UV) \\ M1 (under 365-nm UV) \\ $-\square-\mathrm{MO}$ (under 365-nm UV)}

MO (under day-light)

(b)

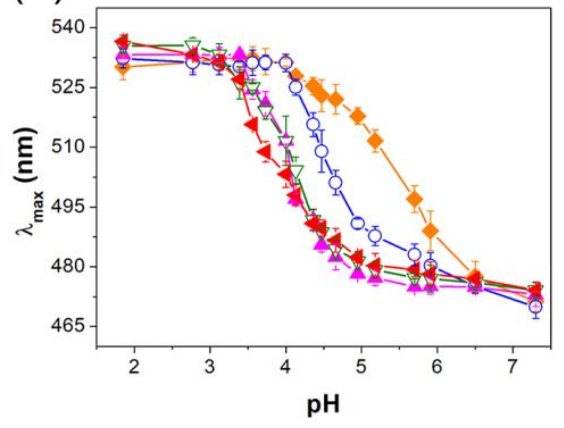

(e)

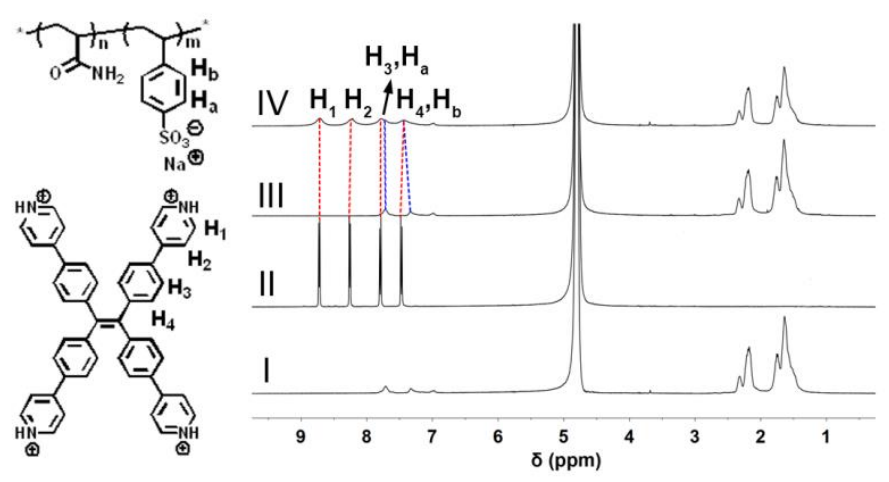

(C)

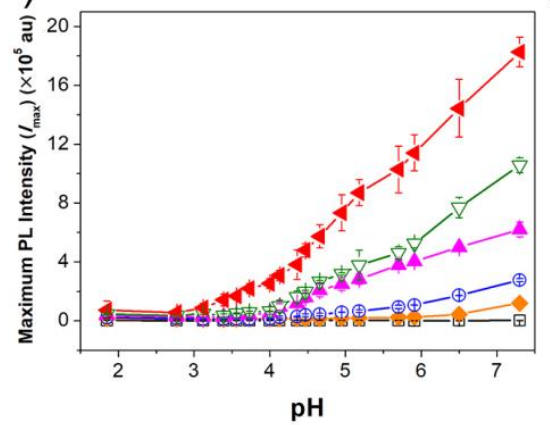

(d)
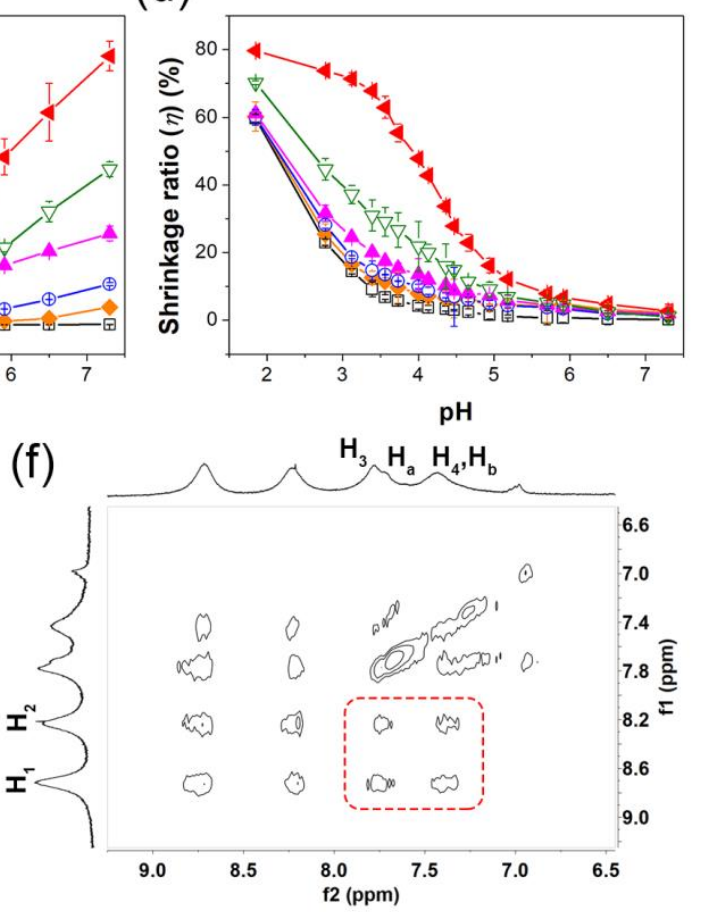

Figure 3. (a) Photographs of monolayer hydrogel samples Mo to $\mathbf{M}$ after immersed in aqueous solutions with different $\mathrm{pH}$ values for $17 \mathrm{~h}$ under 365-nm UV illumination. (The $\mathrm{pH}$ values of every hydrogel samples from left to right are 1.85, 2.77, 3.12, 3.39, $3.56,3.73,4.00,4.13,4.36,4.47,4.66,4.95,5.18,5.70,5.91,6.50$ and 7.30 respectively.) (b) $\lambda_{\max }$ of monolayer hydrogel samples as a function of $\mathrm{pH}$ after immersed in aqueous solutions with different $\mathrm{pH}$ values for $17 \mathrm{~h}$. (c) Maximum PL intensity ( $\left.I_{\mathrm{max}}\right)$ of the monolayer hydrogel samples as a function of $\mathrm{pH}$. (d) Shrinkage ratio $(\eta)$ of the monolayer hydrogel samples as a function of $\mathrm{pH}$. (e) Molecular structures of copolymer network chain of monolayer hydrogel and $4 \mathrm{H}-\mathrm{TPE}-4 \mathrm{Py}^{4+}$, and ${ }^{1} \mathrm{H}$ NMR spectra of (I) a solution of model copolymer PAAm-r-PSS $\left(6.00 \times 10^{-3} \mathrm{mM}\right)$ with $\mathrm{pH}$ of 2.0, (II) a solution of TPE-4Py (1.28 mM) with pH of 2.0, (III) a solution of PAAm-r-PSS $\left(6.00 \times 10^{-3} \mathrm{mM}\right)$ and TPE-4Py $(1.28 \mathrm{mM})$ with $\mathrm{pH}$ of 7.0 , and (IV) a solution of PAAm-r-PSS (6.oo $\left.\times 10^{-3} \mathrm{mM}\right)$ and TPE-4Py $(1.28 \mathrm{mM})$ with $\mathrm{pH}$ of 2.o. (f) Partial $2 \mathrm{D}$ NOESY NMR spectrum of the solution of PAAm- $r$-PSS $(6.00 \times$ $\left.10^{-3} \mathrm{mM}\right)$ and TPE-4Py $(1.28 \mathrm{mM})$ with $\mathrm{pH}$ of 2.0.

value of the mixture was decreased to 2.0, TPE-4Py protonated into ${ }_{4} \mathrm{H}-\mathrm{TPE}-4 \mathrm{Py}^{4+}$ and dissolved, and proton signals of ${ }_{4} \mathrm{H}-\mathrm{TPE}-4 \mathrm{Py}^{4+}$ appeared in the ${ }^{1} \mathrm{H}$ NMR spectrum in Figure ze (IV). However, the shapes and the chemical shifts of the signals change compared with that in Figure $3 e$ (II). The peaks of protons on $4 \mathrm{H}-\mathrm{TPE}-4 \mathrm{Py}^{4+}$ broaden largely and upfield shift when PAAm-r-PSS was added. The broadness is related to the dynamics of phenyl and protonated pyridyl groups on ${ }_{4} \mathrm{H}-\mathrm{TPE}-4 \mathrm{Py}^{4+}$. When ${ }_{4} \mathrm{H}-\mathrm{TPE}-4 \mathrm{Py}^{4+}$ forms electrostatic interactions with PAAm-r-PSS, the movements of the phenyl and protonated pyridyl groups on ${ }_{4} \mathrm{H}-\mathrm{TPE}-4 \mathrm{Py}^{4+}$ are restricted and the rotation of the groups reduces. ${ }^{82}$ Thus, the proton signals become broad. Besides, the interaction with the benzene- 
(a)
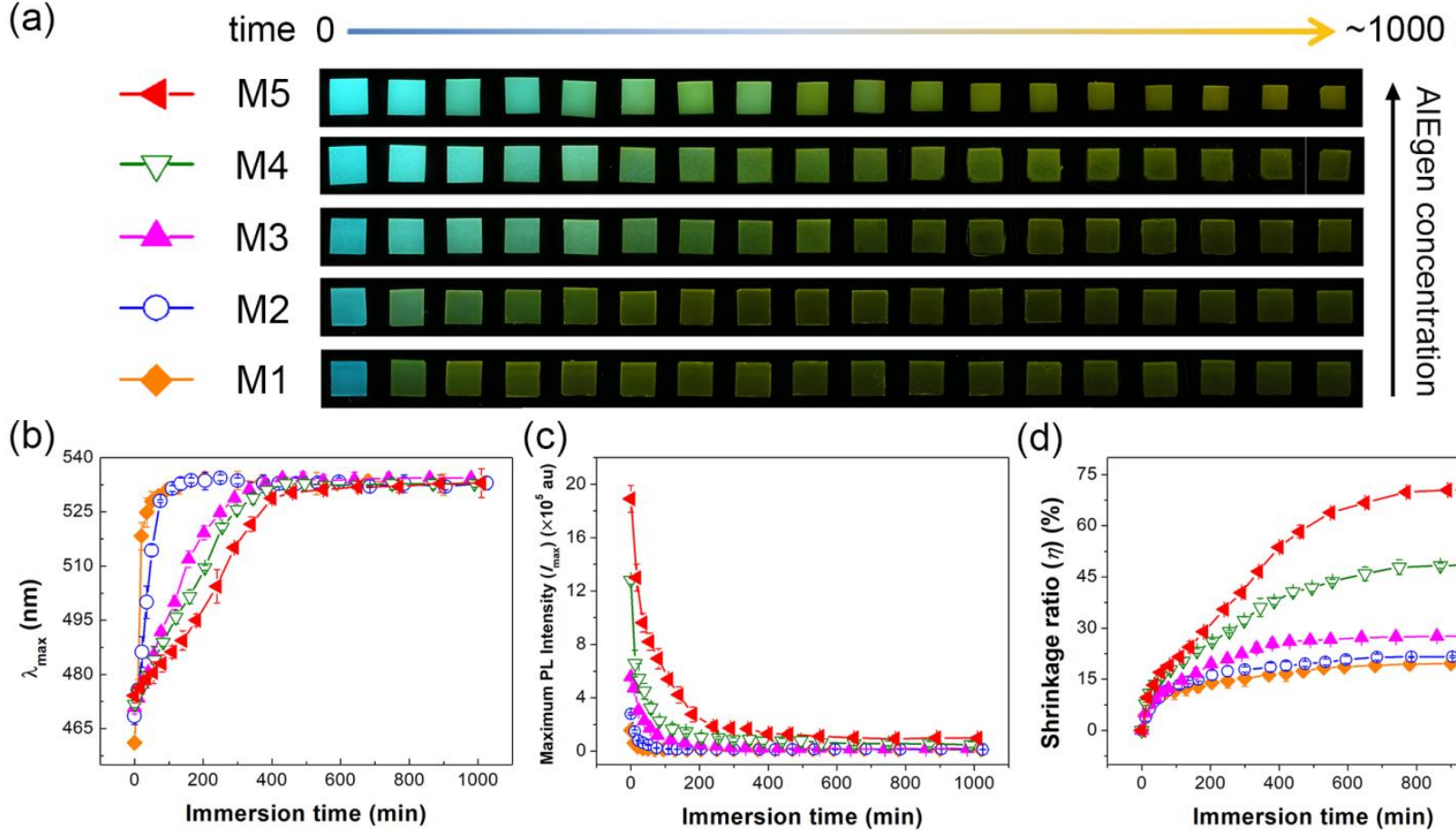

(c)

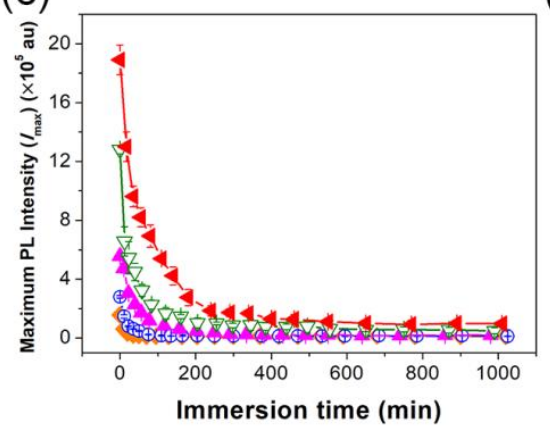

(d)

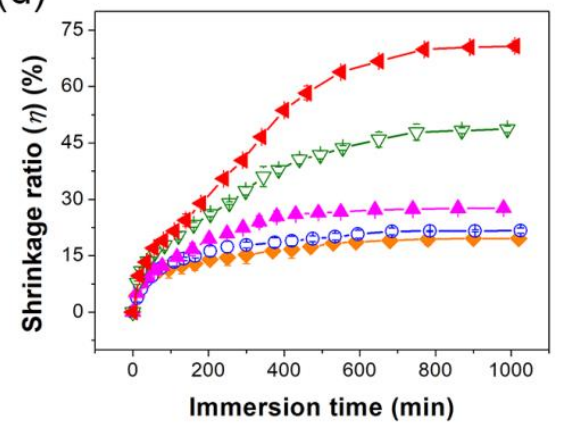

Figure 4. (a) Photographs of monolayer hydrogel samples Mi to M5 after immersed in pH 3.12 aqueous solution with different time under 365-nm UV illumination. (The immersion time of every hydrogel samples from left to right are about o, 15, 35, 55, 8o, $110,140,180,240,300,350,400,450,550,650,750,900$ and 1000 min respectively.) (b) $\lambda_{\max }$ of monolayer hydrogel samples as a function of immersion time after immersed in $\mathrm{pH} 3.12$ aqueous solution. (c) $I_{\max }$ of monolayer hydrogel samples as a function of immersion time after immersed in $\mathrm{pH} 3.12$ aqueous solution. (d) $\eta$ of monolayer hydrogel samples as a function of immersion time after immersed in $\mathrm{pH}_{3.12}$ aqueous solution.

sulfonate groups on PAAm-r-PSS leads to the shielding effect on ${ }_{4} \mathrm{H}-\mathrm{TPE}-4 \mathrm{Py}^{4+}$ protons, which causes their signal to upfield shift. ${ }^{83}$ On the other hand, signals of protons $\mathrm{H}_{\mathrm{a}}$ and $\mathrm{H}_{\mathrm{b}}$ on the benzenesulfonate group on PAAm-r-PSS shift downfield in Figure ze (IV) compared with that in Figure ze (III). This is caused by the interaction with the protonated pridyl groups on $4 \mathrm{H}-\mathrm{TPE}-4 \mathrm{Py}^{4+}$. $2 \mathrm{D}$ NOESY ${ }^{1} \mathrm{H}$ NMR spectra of the $\mathrm{D}_{2} \mathrm{O}$ solution containing both PAAm$r$-PSS and TPE-4Py with the $\mathrm{pH}$ value of 2.0 was also taken. From the spectrum (Figure $3 \mathrm{f}$ ) it can be seen that signals of protons on protonated pridyl groups in $4 \mathrm{H}$-TPE${ }_{4} \mathrm{Py}^{4+}$ correlate with the resonance of benzenesulfonate protons on PAAm-r-PSS. The correlation peaks can not found in the spectrum of the $\mathrm{D}_{2} \mathrm{O}$ solution of TPE-4Py with the $\mathrm{pH}$ value of 2.0 (Figure $\mathrm{S}_{4}$ ). The above NMR results all indicate that electrostatic interactions exist between ${ }_{4} \mathrm{H}-\mathrm{TPE}-4 \mathrm{Py}^{4+}$ and benzenesulfonate groups on the hydrogel network chains.

Dynamic Feature of pH-Responsive Fluorescence Color, Brightness and Volume Changing Properties of TPE-4Py/PAS-Based Monolayer Hydrogels. After the study of the $\mathrm{pH}$-responsiveness of fluorescence color, brightness and volume of TPE-4Py/PASbased monolayer hydrogels in different $\mathrm{pH}$ values, 3.12 was selected as the $\mathrm{pH}$ value to investigate the dynamic process of the fluorescence color, brightness and volume changing of the hydrogels. Swollen hydrogel sheets of M1 to $\mathrm{M}_{5}$ with the size of about $10 \mathrm{~mm} \times 10 \mathrm{~mm}$ were immersed in aqueous solutions with the $\mathrm{pH}$ value of 3.12 .
Photographs under 365-nm UV illumination, fluorescent emission spectra (Figure $\mathrm{S}_{13}$ ) and masses of the hydrogel sheets at different time were measured. Time dependences of $\lambda_{\max }$ and $I_{\max }$ were depicted in Figure $4 \mathrm{~b}$ and c. It can be seen from the photographs (Figure 4a) and the figures that for all hydrogels with the increase of the immersion time, the fluorescent emission red shifts and the fluorescent intensity decreases. These were caused by the protonation of TPE-4Py and the EIR effect of protonated TPE${ }_{4} \mathrm{Py}$. With the increase of TPE-4Py concentration from M1 to $\mathbf{M}_{5}$, the time which the hydrogels spend to reach the equilibrated $\lambda_{\max }$ values about $533 \mathrm{~nm}$ and the equilibrated $I_{\max }$ values increases. With the increase of the amount of TPE-4Py molecules in the hydrogel sheet, the time that the molecules spend to protonate to ${ }_{4} \mathrm{H}-\mathrm{TPE}-4 \mathrm{Py}^{4+}$ increases. Meanwhile, $\eta$ of all hydrogel sheets increases with the increase of the immersion time, and the time which the hydrogel sheets spend to reach the equilibrated $\eta$ values increases with the increase of TPE-4Py concentration from Mi to $\mathbf{M}_{5}$ in Figure $4 \mathrm{~d}$. With the increase of the immersion time, the amount of physical cross-linking points (protonated TPE-4Py) increases, and the hydrogel shrink gradually. With the increase of the amount of TPE${ }_{4} \mathrm{Py}$ molecules in the hydrogel sheet, the time that the molecules spend to protonate to ${ }_{4} \mathrm{H}-\mathrm{TPE}-4 \mathrm{Py}^{4+}$ and form electrostatic interactions with hydrogel network chains increases. Because changes of the fluorescent emission wavelength, the fluorescent intensity and the volume of the monolayer hydrogels root in one element, TPE-4Py, 
(a)

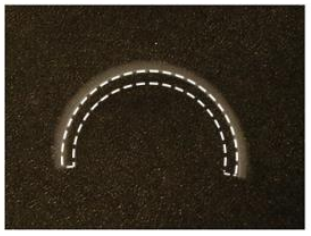

D1

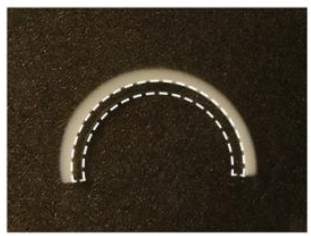

D3

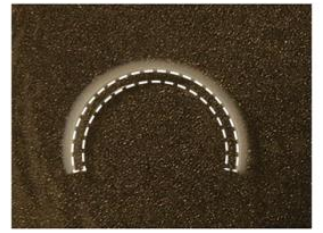

D2

$-\mathrm{O}$

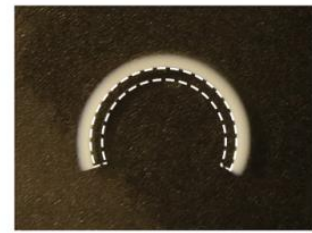

D4

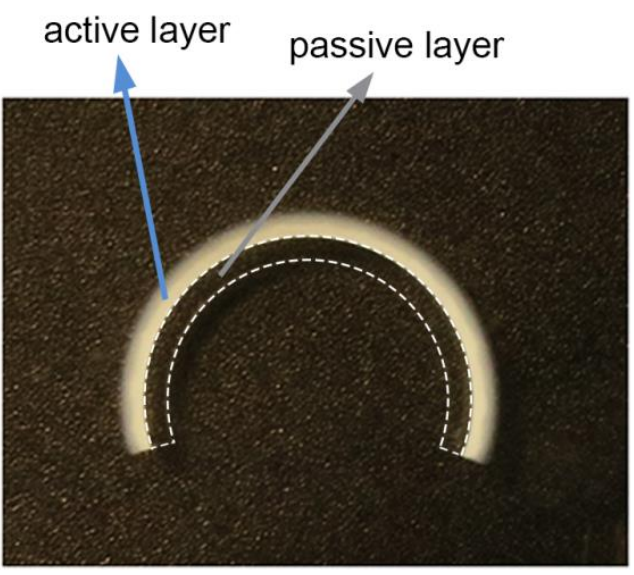

D5

(b) time 0

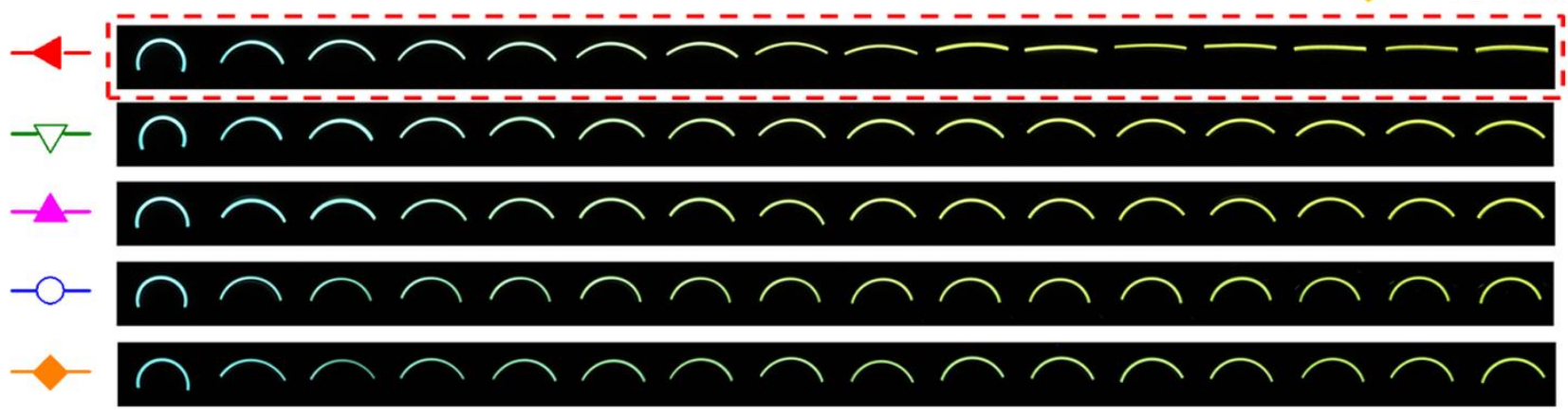

(c)

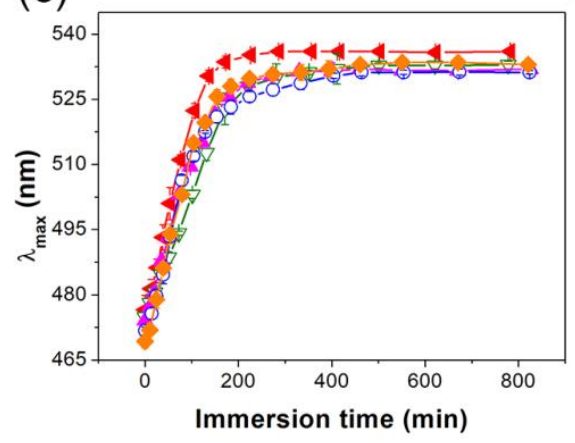

(d)

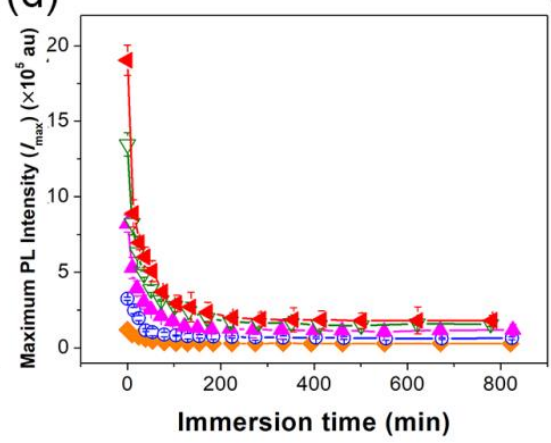

(e)

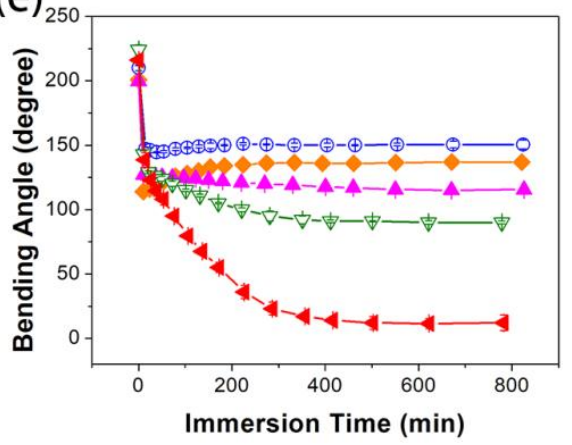

Figure 5. (a) Photographs of fully swollen bilayer hydrogel actuator samples D1 to $\mathbf{D}_{5}$ under day-light. (b) Photographs of the monolayer hydrogel samples after immersed in $\mathrm{pH} 3.12$ aqueous solution with different time under 365-nm UV illumination. (The immersion time of every hydrogel samples from left to right are about o, 12, 25, 38, 53, 77, 105, 135, 170, 225, 285, 355, 415, 500, 625 , and $780 \mathrm{~min}$.) (c) $\lambda_{\max }$ of the monolayer hydrogel samples as a function of immersion time after immersed in $\mathrm{pH} 3.12$ aqueous solution. (d) $I_{\max }$ of the monolayer hydrogel samples as a function of immersion time after immersed in pH 3.12 aqueous solution. (e) Bending angle of the monolayer hydrogel samples as a function of immersion time after immersed in $\mathrm{pH} 3.12$ aqueous solution.

the changes of the three properties are simultaneous and synchronous from Figure 4 .

Dynamic pH-Responsive Fluorescence Color, Brightness Changing and Shape Deformation Properties of TPE-4Py/PAS-Based Bilayer Hydrogel Actuators. To transform the isotropic shrinkage of the monolayer hydrogels into anisotropic $3 \mathrm{D}$ shape deformation, bilayer hydrogel actuators were fabricated through a simple strategy of adding a PAS hydrogel layer without TPE-4Py (Mo) onto the TPE-4Py/PAS-based monolayer hydrogels (Mi to $\mathbf{M}_{5}$ ). The TPE-4Py/PASbased monolayer hydrogel acts as the role of the active layer and the PAS-based monolayer hydrogel acts as the role of the passive layer (Figure $\mathrm{S}_{5}$ ). Thus, two layers of the formed actuators are based on one hydrogel matrix of PAS with the difference that whether the function element TPE-4Py exists. Five bilayer hydrogel actuators Di to $D_{5}$ were prepared from M1 to M5 and Mo (Table S2) according to the procedure in the Supporting Information. 
The as-prepared bilayer hydrogel actuators of D1 to $D_{5}$ were cut into strips with the size of $15 \mathrm{~mm} \times 2 \mathrm{~mm}$, and were immersed in bulk DI water for $12 \mathrm{~h}$. The equilibratedly swollen actuator strips are arc-shaped (Figure 5a) with the transparent passive layer at the inner layer and the white active layer at the outer layer. Auxiliary white dash lines were depicted in the photographs to facilitate the recognition of the passive layers.

The arc-shaped bilayer hydrogel actuator strips were immersed in aqueous solutions with the $\mathrm{pH}$ value of 3.12. Photographs under 365-nm UV illumination, fluorescent emission spectra (Figure S14) and bending angles of them at different time were measured. The fluorescent emission spectra were measured from the direction of the passive layer. Time dependences of $\lambda_{\max }$ and $I_{\max }$ were depicted in Figure $5 \mathrm{c}$ and $\mathrm{d}$. It can be seen from the photographs (Figure 5b) and the figures that for all actuator strips with the increase of the immersion time, the fluorescent emission red shifts and the fluorescent intensity decreases. These were caused by the protonation of TPE-4Py and the EIR effect of protonated TPE-4Py in the active layer. With the increase of the TPE-4Py concentration in the active layer of the actuator strips from $D_{1}$ to $D_{5}$, the time which the hydrogels spend to reach the equilibrated $\lambda_{\max }$ values and the equilibrated $I_{\max }$ values tends to be the same. This character is different from that of the monolayer hydrogels Mi to M5. The fluorescent emission spectra were measured from the direction of the passive layer. Thus the spectra showed the fluorescent properties of TPE-4Py located at the interface between the active and the passive layers. The protonated TPE-4Py molecules could migrate into the passive layer in short distance and form electrostatic interactions with the network chains of the passive layer. These protonated TPE-4Py molecules mainly contribute to the fluorescent emission spectra of the bilayer hydrogel actuators. Under this circumstance, the migration of the protonated TPE-4Py molecules is the key factor to determine the dynamics of the changing of the fluorescent properties of the actuators. The migration rates of the protonated $\mathrm{TPE}-4 \mathrm{Py}$ are consistent in all bilayer hydrogels. Thus the dynamics of the fluorescent properties changing of D1 to $D_{5}$ are similar. Meanwhile, after the arc-shaped bilayer hydrogel actuator strips were immersed in aqueous solutions with the $\mathrm{pH}$ value of 3.12, the strips gradually unbent (Figure $5 \mathrm{~b}$ ). Time dependences of bending angles of the strips were depicted in Figure 5e. It can be seen from the figure that with the increase of the immersion time, bending angles of all strips of D1 to $D_{5}$ decrease gradually and finally reach equilibriums. With the increase of the immersion time, the increase of the amount of physical cross-linking points (protonated TPE${ }_{4} \mathrm{Py}$ ) causes the active layer to shrink, and the generated force at the interface between the active and the passive layers causes the actuator strips to unbend. With the increase of the immersion time, bending angles of actuator strips D1 and D2 first quickly decrease, then increase a little, and finally reach equilibriums. After the actuator strips were immersed in the $\mathrm{pH} 3.12$ aqueous solution, the TPE-4Py first protonated and formed electrostatic inter- actions with network chains of the active layer. These newly formed physical cross-linking points resulted in the shrinkage of the active layer and the unbending of the strips. Then a small amount of protonated TPE-4Py molecules migrated into the passive layer and acted as the physical cross-linking points to interact with its network chains. This caused the passive layer to shrink slightly, and the strips recovered slightly. When the TPE-4Py concentration in the active layer further increases, this bending angle recovery phenomenon disappears for $\mathbf{D}_{\mathbf{3}}$ to $\mathbf{D}_{\mathbf{5}}$. The newly formed protonated TPE-4Py could either form interactions with network chains in the active layer or migrate and form interactions with network chains in the passive layer. Nevertheless, the amount of the migrated protonated $\mathrm{TPE}_{4} \mathrm{Py}$ molecules is small and the majority of the protonated TPE-4Py molecules act as the physical cross-linking points in the active layer. When the TPE${ }_{4} \mathrm{Py}$ concentration increases in the active layer, the proportion of the amount of the migrated protonated TPE${ }_{4} \mathrm{Py}$ molecules in all protonated ones decreases, and the recovery effect can not be appeared. With the increase of the TPE-4Py concentration in the actuator strips from $\mathbf{D}_{3}$ to $\mathbf{D}_{5}$, the time which the strips spend to reach equilibriums increases. With the increase of the amount of TPE${ }_{4} \mathrm{Py}$ molecules in the strips, the time that the molecules spend to protonate to $4 \mathrm{H}-\mathrm{TPE}-4 \mathrm{Py}^{4+}$ and form electrostatic interactions with hydrogel network chains increases. From Figure $5 \mathrm{~b}$ to e, it can be seen that the changes of the fluorescent emission wavelength, the fluorescent intensity and the bending angle of the bilayer hydrogel actuators are simultaneous and synchronous. This is profited from the core role of TPE-4Py as the origin of the changes of the three properties. Because the bilayer hydrogel actuator strip D5 $_{5}$ had the moderate changing rate of fluorescence color, brightness and curvature, it was selected to prepare the complex-shaped actuator and simulate the blooming process of natural flowers with the ability of simultaneous color changing during blooming.

A flower-shaped bilayer hydrogel actuator based on $\mathrm{D}_{5}$ was prepared through cutting of the as-prepared $D_{5}$ bilayer hydrogel film. After the actuator was immersed in DI water to swell to the equilibrium, it turned into furling state. Then it was immersed in the aqueous solution with $\mathrm{pH}$ value of 3.12. Photographs of the actuator were taken every several minutes until 780 min from side and top views under 365-nm UV illumination. 18 photographs were selected to show the process of simultaneous fluorescence color and brightness changes and complex shape deformation of the actuator in Figure 6. It can be seen that with the increase of the immersion time, the artificial flower made by $\mathbf{D}_{5}$ gradually blooms accompanied with simultaneous fluorescence color and brightness changes. All the photographs are also made into two movies (Movie $\mathrm{M}_{1}$ and $\mathrm{M}_{2}$ ).

AIE Effect during the Process of the Simultaneous Fluorescence Color, Brightness and Shape Changing of Bilayer Hydrogel Actuators. In the neutral medium, the origin of the fluorescence of the actuator is aggregated TPE-4Py encapsulated in the active layer. 

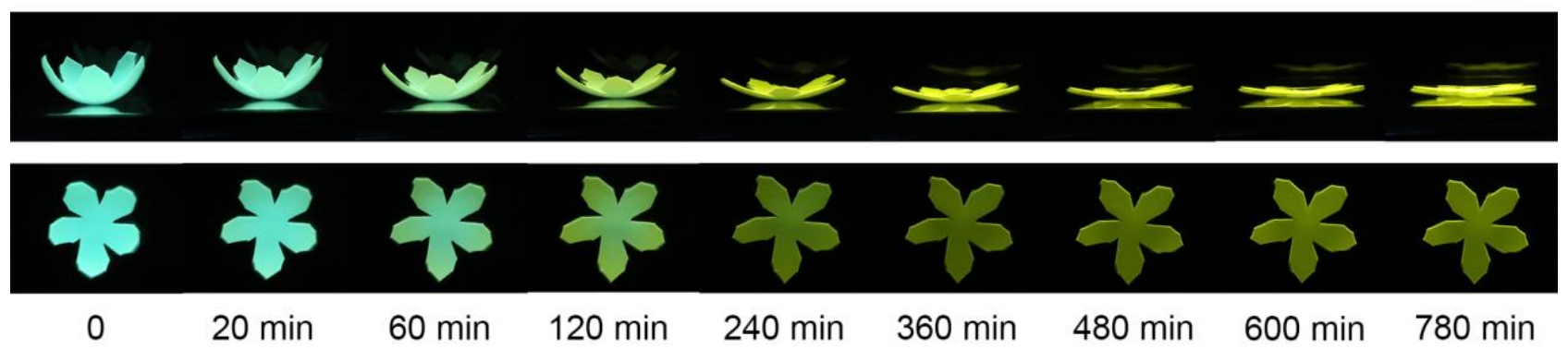

Figure 6. Simultaneous emission change and complex shape deformation of $\mathbf{M}_{\mathbf{5}}$ based hydrogel actuator. (Photographs were taken under $365-\mathrm{nm}$ UV illumination. The upper line is flat view and the lower line is plane view.)

AIE effect causes the TPE-4Py to emit strong sapphire light. TPE-4Py forms aggregates in the neutral water and emits strong fluorescence. When TPE-4Py protonates to ${ }_{4} \mathrm{H}-\mathrm{TPE}-4 \mathrm{Py}^{4+}$ in the aqueous solution, the dissolution of ${ }_{4} \mathrm{H}-\mathrm{TPE}-4 \mathrm{Py}^{4+}$ activates its intromolecular rotation. Thus the red shifted fluorescent light of ${ }_{4} \mathrm{H}-\mathrm{TPE}-4 \mathrm{Py}^{4+}$ is pretty weak. The maximum PL intensity of the TPE-4Py suspension with the $\mathrm{pH}$ value of $6.98\left(I_{\mathrm{pH}=6.98}\right)$ is 221.5 times that of the ${ }_{4} \mathrm{H}$-TPE-4Py ${ }^{4+}$ solution with the $\mathrm{pH}$ value of 3.34 $\left(I_{\mathrm{pH}=3.34}\right)$ (Figure $\left.\mathrm{S} 16\right)$. Quantum yields (QYs) of the TPE${ }_{4} \mathrm{Py}$ suspensions with different $\mathrm{pH}$ values were also measured (Figure $\mathrm{S}_{15 \mathrm{a}}$ ), and QY of TPE-4Py suspension with the $\mathrm{pH}$ value of $6.98\left(\mathrm{QY}_{\mathrm{pH}=6.98}\right)$ is 8.6 times that of ${ }_{4} \mathrm{H}$ TPE-4 $\mathrm{Py}^{4+}$ solution with the $\mathrm{pH}$ value of $3.34\left(\mathrm{QY}_{\mathrm{pH}=3.34}\right)$. However, for the TPE-4Py/PAS-based monolayer hydrogels and bilayer hydrogel actuators, the differences of fluorescence brightness before and after immersed in $\mathrm{pH}$ 3.12 aqueous solution are largely reduced. The maximum PL intensity of $\mathbf{D}_{5}$ before the immersion ( $I_{\text {before immersion }}$ ) is only 10.5 times that of it after the immersion ( $I_{\text {after immersion }}$ ), and QY of $D_{5}$ before the immersion ( $\left.Q_{\text {before immersion }}\right)$ is only 2.3 times that of it after the immersion $\left(\mathrm{QY}_{\text {after immer- }}\right.$ sion). After the protonation of TPE-4Py, the formed $4 \mathrm{H}$ TPE-4 $\mathrm{Py}^{4+}$ electrostatically interacts with network chains of the hydrogel. These interactions and the network polymer chains largely restrict the intramolecular rotation of ${ }_{4} \mathrm{H}-\mathrm{TPE}-4 \mathrm{Py}^{4+}$, which leads to the visible red shifted fluorescent emission.

\section{CONCLUSIONS}

In summary, bilayer hydrogel actuators with abilities of simultaneous fluorescence color and brightness changes and complex shape deformation under one stimulus were designed and fabricated using TPE-4Py as the core function element and PAS as the matrix. TPE-4Py aggregates encapsulated PAS hydrogel acted as the active layer of the actuators and blank PAS hydrogel acted as the passive layer. TPE-4Py/PAS-based monolayer hydrogels and bilayer hydrogel actuators with different TPE-4Py concentration were prepared and their $\mathrm{pH}$-responsiveness was investigated. When the flower-shaped actuator $\mathbf{M}_{5}$ were immersed in aqueous solution with $\mathrm{pH}$ value of 3.12, it could simultaneously change its fluorescence color, brightness and shape during $13 \mathrm{~h}$. Protonation of TPE-4Py led to fluorescence color and brightness changes of the actuators and meanwhile electrostatic interactions occurred between the formed protonated TPE-4Py and benzenesulfonate groups on PAS chains in the active layer caused the actuators to deform. The strategy of combining AIEgens with bilayer hydrogels to construct stimuliresponsive hydrogels with complex practical functions in this work provides new insights in the design of intelligent systems. The prepared TPE-4Py/PAS-based bilayer hydrogel actuators are potential to be applied in hightech fields such as ${ }_{3} \mathrm{D}$ and ${ }_{4} \mathrm{D}$ printing, soft robots, smart wearable devices and drug delivery systems.

\section{ASSOCIATED CONTENT}

Supporting Information.

Experimental Section and additional information related to the Results and Discussion section (PDF)

Movies M1 and M2

\section{AUTHOR INFORMATION}

\section{Corresponding Author}

*chjacky@ust.hk

*tangbenz@ust.hk

Notes

The authors declare no competing financial interest.

\section{ACKNOWLEDGMENT}

J. W. Y. L. and B.Z.T. acknowledge the financial support from the National Science Foundation of China (21788102, 21490570 and 21490574), the Research Grant Council of Hong Kong (16308116, 16305618 and C6009-17G), the Science and Technology Plan of Shenzhen (JCYJ20160229205601482, JCY20170307173739739 and JCYJ20170818113602462) and the Innovation and Technology Commission (ITC-CNERC149C01).

\section{REFERENCES}

(1) Schull, J. Are Species Intelligent? Behav. Brain Sci. 1990, $13(1), 63-75$.

(2) van Loon, L. C. The Intelligent Behavior of Plants. Trends Plant Sci. 2016, 21 (4), 286-294.

(3) Teyssier, J.; Saenko, S. V.; van der Marel, D.; Milinkovitch, M. C. Photonic crystals cause active colour change in chameleons. Nat. Commun. 2015, 6, 6368. 
(4) Crookes, W. J.; Ding, L.-L.; Huang, Q. L.; Kimbell, J. R.; Horwitz, J.; McFall-Ngai, M. J. Reflectins: The Unusual Proteins of Squid Reflective Tissues. Science 2004, 303 (5655), 235-238.

(5) Wilker, J. J. How to suck like an octopus. Nature 2017, $546,358-359$.

(6) Shine, R. Function and evolution of the frill of the frillneck lizard, Chlamydosaurus kingii (Sauria: Agamidae). Biol. J. Linn. Soc. 2008, 40 (1), 11-20.

(7) Hamilton, D. G.; Whiting, M. J.; Pryke, S. R. Fiery frills: carotenoid-based coloration predicts contest success in frillneck lizards. Behav. Ecol. 2013, 24 (5), 1138-1149.

(8) Vaknin, H.; Bar-Akiva, A.; Ovadia, R.; Nissim-Levi, A.; Forer, I.; Weiss, D.; Oren-Shamir, M. Active anthocyanin degradation in Brunfelsia calycina (yesterday-today-tomorrow) flowers. Planta 2005, 222 (1), 19-26.

(9) Li, M.; Sun, Y. T.; Lu, X. C.; Debnath, B.; Mitra, S.; Qiu, D. L. Proteomics Reveal the Profiles of Color Change in Brunfelsia acuminata Flowers. Int. J. Mol. Sci. 2019, 20 (8), 200o2016.

(10) Eisikowitch, D.; Rotem, R. Flower Orientation and Color Change in Quisqualis Indica and Their Possible Role in Pollinator Partitioning. Bot. Gaz. 1987, 148 (2), 175-179.

(11) Yan, J.; Wang, M.; Zhang, L. Light induces petal color change in Quisqualis indica (Combretaceae). Plant Diversity 2018, 40 (1), 28-34.

(12) Pereira, A. C.; da Silva, J. B.; Goldenberg, R.; Melo, G. A. R.; Varassin, I. G. Flower color change accelerated by bee pollination in Tibouchina (Melastomataceae). Flora 2011, 206 (5), 491-497.

(13) Weiss, M. R. Floral Color Changes as Cues for Pollinators. Nature 1991, 354 (6350), 227-229.

(14) Wang, H.; Ji, X. F.; Li, Z. T.; Huang, F. H. Fluorescent Supramolecular Polymeric Materials. Adv. Mater. 2017, 29 (14), 1606117.

(15) Zhang, X.; Chen, L. F.; Lim, K. H.; Gonuguntla, S.; Lim, K. W.; Pranantyo, D.; Yong, W. P.; Yam, W. J. T.; Low, Z.; Teo, W. J.; Nien, H. P.; Loh, Q. W.; Soh, S. The Pathway to Intelligence: Using Stimuli-Responsive Materials as Building Blocks for Constructing Smart and Functional Systems. Adv. Mater. 2019, 31 (11), 1804540

(16) Cobo, I.; Li, M.; Sumerlin, B. S.; Perrier, S. Smart hybrid materials by conjugation of responsive polymers to biomacromolecules. Nat. Mater. 2015, 14 (2), 143-159.

(17) Yu, X. W.; Cheng, H. H.; Zhang, M.; Zhao, Y.; Qu, L. T.; Shi, G. Q. Graphene-based smart materials. Nat. Rev. Mater. 2017, 2 (9), 17046.

(18) Xu, W. N.; Kwok, K. S.; Gracias, D. H. Ultrathin Shape Change Smart Materials. Accounts Chem. Res. 2018, 51 (2), 436444.

(19) Doring, A.; Birnbaum, W.; Kuckling, D. Responsive hydrogels - structurally and dimensionally optimized smart frameworks for applications in catalysis, micro-system technology and material science. Chem. Soc. Rev. 2013, 42 (17), 7391-7420.

(20) Ji, X. F.; Huang, F. H. A rapidly self-healing supramolecular polymer hydrogel. Sci. China-Chem. 2015, 58 (3), 436-437.

(21) Ma, C.; Le, X.; Tang, X.; He, J.; Xiao, P.; Zheng, J.; Xiao, H.; Lu, W.; Zhang, J.; Huang, Y.; Chen, T. A Multiresponsive Anisotropic Hydrogel with Macroscopic ${ }_{3} \mathrm{D}$ Complex Deformations. Adv. Funct. Mater. 2o16, 26 (47), 8670-8676.

(22) Wei, S.; Lu, W.; Le, X.; Ma, C.; Lin, H.; Wu, B.; Zhang, J.; Theato, P.; Chen, T. Bioinspired Synergistic FluorescenceColor Switchable Polymeric Hydrogel Actuator. Angew. Chem., Int. Edit. 2019. DOI: 10.1002/anie.201908437.
(23) Ilmain, F.; Tanaka, T.; Kokufuta, E. Volume transition in a gel driven by hydrogen bonding. Nature 1991, 349 (6308), 400-401.

(24) Xia, L. W.; Xie, R.; Ju, X. J.; Wang, W.; Chen, Q. M.; $\mathrm{Chu}, \mathrm{L}$. Y. Nano-structured smart hydrogels with rapid response and high elasticity. Nat. Commun. 2013, 4, 2226.

(25) Zhang, X. N.; Wang, Y. J.; Sun, S.; Hou, L.; Wu, P.; Wu, Z. L.; Zheng, Q. A Tough and Stiff Hydrogel with Tunable Water Content and Mechanical Properties Based on the Synergistic Effect of Hydrogen Bonding and Hydrophobic Interaction. Macromolecules 2018, 51 (20), 8136-8146.

(26) Lee, K.; Asher, S. A. Photonic Crystal Chemical Sensors: pH and Ionic Strength. J. Am. Chem. Soc. 2ooo, 122 (39), 9534-9537.

(27) Ma, C.; Li, T.; Zhao, Q.; Yang, X.; Wu, J.; Luo, Y.; Xie, T. Supramolecular Lego Assembly Towards Three-Dimensional Multi-Responsive Hydrogels. Adv. Mater. 2014, 26 (32), 56655669 .

(28) Mamada, A.; Tanaka, T.; Kungwatchakun, D.; Irie, M. Photoinduced phase transition of gels. Macromolecules 1990, 23 (5), 1517-1519.

(29) Wang, H.; Zhu, C. N.; Zeng, H.; Ji, X. F.; Xie, T.; Yan, X. Z.; Wu, Z. L.; Huang, F. H. Reversible Ion-Conducting Switch in a Novel Single-Ion Supramolecular Hydrogel Enabled by Photoresponsive Host-Guest Molecular Recognition. Adv. Mater. 2019, 31 (12), 1807328.

(30) Tanaka, T.; Nishio, I.; Sun, S. T.; Uenonishio, S. Collapes of Gels in An Electric-Field. Science 1982, 218 (4571), 467-469.

(31) Hu, K.; Sun, J.; Guo, Z.; Wang, P.; Chen, Q.; Ma, M.; Gu, N. A Novel Magnetic Hydrogel with Aligned Magnetic Colloidal Assemblies Showing Controllable Enhancement of Magnetothermal Effect in the Presence of Alternating Magnetic Field. Adv. Mater. 2015, 27 (15), 2507-2514.

(32) Zheng, S. Y.; Shen, Y.; Zhu, F.; Yin, J.; Qian, J.; Fu, J.; $\mathrm{Wu}, \mathrm{Z}$. L.; Zheng, Q. Programmed Deformations of $3 \mathrm{D}$-Printed Tough Physical Hydrogels with High Response Speed and Large Output Force. Adv. Funct. Mater. 2018, 28 (37), 1803366.

(33) Tu, T.; Fang, W.; Sun, Z. Visual-Size Molecular Recognition Based on Gels. Adv. Mater. 2013, 25 (37), 5304-5313.

(34) Jia, H.; Li, Z.; Wang, X.; Zheng, Z. Facile functionalization of a tetrahedron-like PEG macromonomerbased fluorescent hydrogel with high strength and its heavy metal ion detection. J. Mater. Chem. A 2015, 3 (3), 1158-1163.

(35) Stoychev, G.; Zakharchenko, S.; Turcaud, S.; Dunlop, J. W. C.; Ionov, L. Shape-Programmed Folding of StimuliResponsive Polymer Bilayers. ACS Nano 2012, 6 (5), 3925-3934.

(36) Yao, C.; Liu, Z.; Yang, C.; Wang, W.; Ju, X. J.; Xie, R.; Chu, L. Y. Poly(N-isopropylacrylamide)-Clay Nanocomposite Hydrogels with Responsive Bending Property as TemperatureControlled Manipulators. Adv. Funct. Mater. 2015, 25 (20), 29802991.

(37) Stoychev, G.; Guiducci, L.; Turcaud, S.; Dunlop, J. W. C.; Ionov, L. Hole-Programmed Superfast Multistep Folding of Hydrogel Bilayers. Adv. Funct. Mater. 2016, 26 (42), 7733-7739.

(38) Liu, L.; Ghaemi, A.; Gekle, S.; Agarwal, S. OneComponent Dual Actuation: Poly(NIPAM) Can Actuate to Stable 3D Forms with Reversible Size Change. Adv. Mater. 2016, 28 (44), 9792-9796.

(39) Kim, D.; Kim, H.; Lee, E.; Jin, K. S.; Yoon, J. Programmable Volume Phase Transition of Hydrogels Achieved by Large Thermal Hysteresis for Static-Motion Bilayer Actuators. Chem. Mater. 2016, 28 (23), 8807-8814.

(40) Duan, J.; Liang, X.; Zhu, K.; Guo, J.; Zhang, L. Bilayer hydrogel actuators with tight interfacial adhesion fully constructed from natural polysaccharides. Soft Matter 2017, 13 (2), 345-354. 
(41) Wang, Z. J.; Hong, W.; Wu, Z. L.; Zheng, Q. SiteSpecific Pre-Swelling-Directed Morphing Structures of Patterned Hydrogels. Angew. Chem., Int. Ed. 2017, 56 (50), 15974-15978.

(42) Cheng, Y.; Huang, C.; Yang, D.; Ren, K.; Wei, J. Bilayer hydrogel mixed composites that respond to multiple stimuli for environmental sensing and underwater actuation. J. Mater. Chem. B 2018, 6 (48), 8170-8179.

(43) Gao, G.; Wang, Z.; Xu, D.; Wang, L.; Xu, T.; Zhang, H.; Chen, J.; Fu, J. Snap-Buckling Motivated Controllable Jumping of Thermo-Responsive Hydrogel Bilayers. ACS Appl. Mater. Interfaces 2018, 10 (48), 41724-41731.

(44) Hu, Z.; Zhang, X.; Li, Y. Synthesis and application of modulated polymer gels. Science 1995, 269 (5223), 525-527.

(45) Haque, M. A.; Kamita, G.; Kurokawa, T.; Tsujii, K.; Gong, J. P. Unidirectional Alignment of Lamellar Bilayer in Hydrogel: One-Dimensional Swelling, Anisotropic Modulus, and Stress/Strain Tunable Structural Color. Adv. Mater. 2010, 22 (45), 5110-5114.

(46) Zhang, Q. M.; Xu, W.; Serpe, M. J. Optical Devices Constructed from Multiresponsive Microgels. Angew. Chem., Int. Ed. 2014, 53 (19), 4827-4831.

(47) Görl, D.; Soberats, B.; Herbst, S.; Stepanenko, V.; Würthner, F. Perylene bisimide hydrogels and lyotropic liquid crystals with temperature-responsive color change. Chem. Sci. 2016, 7 (11), 6786-679o.

(48) Lu, W.; Ma, C.; Zhang, D.; Le, X.; Zhang, J.; Huang, Y.; Huang, C.-F.; Chen, T. Real-Time in Situ Investigation of Supramolecular Shape Memory Process by Fluorescence Switching. J. Phys. Chem. C 2018, 122 (17), 9499-9506.

(49) Mei, J.; Leung, N. L. C.; Kwok, R. T. K.; Lam, J. W. Y.; Tang, B. Z. Aggregation-Induced Emission: Together We Shine, United We Soar! Chem. Rev. 2015, 115 (21), 11718-11940.

(50) Luo, J. D.; Xie, Z. L.; Lam, J. W. Y.; Cheng, L.; Chen, H. Y.; Qiu, C. F.; Kwok, H. S.; Zhan, X. W.; Liu, Y. Q.; Zhu, D. B.; Tang, B. Z. Aggregation-induced emission of 1-methyl-1,2,3,4,5pentaphenylsilole. Chem. Commun. 2001(18), 1740-1741.

(51) Mei, J.; Hong, Y.; Lam, J. W. Y.; Qin, A.; Tang, Y.; Tang, B. Z. Aggregation-Induced Emission: The Whole Is More Brilliant than the Parts. Adv. Mater. 2014, 26 (31), 5429-5479.

(52) Zheng, H. Y.; Li, C. Y.; He, C. C.; Dong, Y. Q.; Liu, Q. S.; Qin, P. F.; Zeng, C.; Wang, H. L. Luminescent hydrogels based on di(4-propoxyphenyl)-dibenzofulvene exhibiting four emission colours and organic solvents/thermal dual-responsive properties. J. Mater. Chem. C 2014, 2 (29), 5829-5835.

(53) Wang, Z. K.; Nie, J. Y.; Qin, W.; Hu, Q. L.; Tang, B. Z. Gelation process visualized by aggregation-induced emission fluorogens. Nat. Commun. 2016, 7, 12033.

(54) Yan, X. Z.; Cook, T. R.; Wang, P.; Huang, F. H.; Stang, P. J. Highly emissive platinum(II) metallacages. Nat. Chem. 2015, 7 (4), 342-348.

(55) Hu, R. R.; Kang, Y.; Tang, B. Z. Recent advances in AIE polymers. Polym. J. 2016, 48 (4), 359-370.

(56) Feng, H. T.; Yuan, Y. X.; Xiong, J. B.; Zheng, Y. S.; Tang, B. Z. Macrocycles and cages based on tetraphenylethylene with aggregation-induced emission effect. Chem. Soc. Rev. 2018, 47 (19), 7452-7476.

(57) Qi, J.; Chen, C.; Ding, D.; Tang, B. Z. AggregationInduced Emission Luminogens: Union Is Strength, Gathering Illuminates Healthcare. Adv. Healthcare Mater. 2018, 7 (20), 1800477 .

(58) Ji, X.; Shi, B.; Wang, H.; Xia, D.; Jie, K.; Wu, Z. L.; Huang, F. Supramolecular Construction of Multifluorescent Gels: Interfacial Assembly of Discrete Fluorescent Gels through Multiple Hydrogen Bonding. Adv. Mater. 2015, 27 (48), 8o628066.

(59) Dong, Y. Q.; Lam, J. W. Y.; Qin, A.; Sun, J. X.; Liu, J. Z.; Li, Z.; Sun, J. Z.; Sung, H. H. Y.; Williams, I. D.; Kwok, H. S.; Tang,
B. Z. Aggregation-induced and crystallization-enhanced emissions of 1,2-diphenyl-3,4-bis(diphenylmethylene)-1cyclobutene. Chem. Commun. 2007(31), 3255-3257.

(6o) Lin, Q.; Lu, T.-T.; Zhu, X.; Wei, T.-B.; Li, H.; Zhang, Y.M. Rationally introduce multi-competitive binding interactions in supramolecular gels: a simple and efficient approach to develop multi-analyte sensor array. Chem. Sci. 2016, 7 (8), 53415346.

(61) Cheng, Y.; Wang, J.; Qiu, Z.; Zheng, X.; Leung, N. L. C.; Lam, J. W. Y.; Tang, B. Z. Multiscale Humidity Visualization by Environmentally Sensitive Fluorescent Molecular Rotors. Adv. Mater. 2017, 29 (46), 1703900.

(62) Liu, S.; Cheng, Y.; Zhang, H.; Qiu, Z.; Kwok, R. T. K.; Lam, J. W. Y.; Tang, B. Z. In Situ Monitoring of RAFT Polymerization by Tetraphenylethylene-Containing Agents with Aggregation-Induced Emission Characteristics. Angew. Chem., Int. Ed. 2018, 57 (21), 6274-6278.

(63) Cheng, Y. H.; Liu, S. J.; Song, F. Y.; Khorloo, M.; Zhang, H. K.; Kwok, R. T. K.; Lam, J. W. Y.; He, Z. K.; Tang, B. Z. Facile emission color tuning and circularly polarized light generation of single luminogen in engineering robust forms. Mater. Horizons 2019, 6 (2), 405-411.

(64) Mutai, T.; Tomoda, H.; Ohkawa, T.; Yabe, Y.; Araki, K. Switching of Polymorph-Dependent ESIPT Luminescence of an Imidazo[1,2-a]pyridine Derivative. Angew. Chem., Int. Ed. 20o8, 47 (49), 9522-9524.

(65) Chen, X.; Shen, X. Y.; Guan, E.; Liu, Y.; Qin, A.; Sun, J. Z.; Tang, B. Z. A pyridinyl-functionalized tetraphenylethylene fluorogen for specific sensing of trivalent cations. Chem. Commun. 2013, 49 (15), 1503-1505.

(66) Furukawa, S.; Shono, H.; Mutai, T.; Araki, K. Colorless, Transparent, Dye-Doped Polymer Films Exhibiting Tunable Luminescence Color: Controlling the Dual-Color Luminescence of 2-(2 ' -Hydroxyphenyl)imidazo[1,2-a]pyridine Derivatives with the Surrounding Matrix. ACS Appl. Mater. Interfaces 2014, 6 (18), 16065-16070.

(67) Wang, J. G.; Gu, X. G.; Zhang, P. F.; Huang, X. B.; Zheng, X. Y.; Chen, M.; Feng, H. T.; Kwok, R. T. K.; Lam, J. W. Y.; Tang, B. Z. Ionization and Anion-pi(+) Interaction: A New Strategy for Structural Design of Aggregation-Induced Emission Luminogens. J. Am. Chem. Soc. 2o17, 139 (46), 16974-16979.

(68) Feng, X.; Li, Y.; He, X.; Liu, H.; Zhao, Z.; Kwok, R. T. K.; Elsegood, M. R. J.; Lam, J. W. Y.; Tang, B. Z. A SubstitutionDependent Light-Up Fluorescence Probe for Selectively Detecting $\mathrm{Fe}_{3}+$ Ions and Its Cell Imaging Application. Adv. Funct. Mater. 2018, 28 (35), 1802833.

(69) Sun, Y.; Yao, Y.; Wang, H.; Fu, W.; Chen, C.; Saha, M. L.; Zhang, M.; Datta, S.; Zhou, Z.; Yu, H.; Li, X.; Stang, P. J. SelfAssembly of Metallacages into Multidimensional Suprastructures with Tunable Emissions. J. Am. Chem. Soc. 2018, 140 (40), 12819-12828.

(70) Yan, X.; Wang, F.; Zheng, B.; Huang, F. Stimuliresponsive supramolecular polymeric materials. Chem. Soc. Rev. 2012, 41 (18), 6042-6065.

(71) Wei, P.; Yan, X.; Huang, F. Supramolecular polymers constructed by orthogonal self-assembly based on host-guest and metal-ligand interactions. Chem. Soc. Rev. 2015, 44 (3), 815832.

(72) Wang, M.; Zheng, Y. R.; Ghosh, K.; Stang, P. J. Metallosupramolecular Tetragonal Prisms via Multicomponent Coordination-Driven Template-Free Self-Assembly. J. Am. Chem. Soc. 2010, 132 (18), 6282-6283.

(73) Sun, Y.; Zhang, F.; Jiang, S.; Wang, Z.; Ni, R.; Wang, H.; Zhou, W.; Li, X.; Stang, P. J. Assembly of Metallacages into Soft Suprastructures with Dimensions of up to Micrometers and the Formation of Composite Materials. J. Am. Chem. Soc. 2018, 140 (49), 17297-17307. 
(74) Ji, X.; Li, Z.; Liu, X.; Peng, H.-Q.; Song, F.; Qi, J.; Lam, J. W. Y.; Long, L.; Sessler, J. L.; Tang, B. Z. A Functioning Macroscopic "Rubik's Cube" Assembled via Controllable Dynamic Covalent Interactions. Adv. Mater. 2019, 1902365. DOI : 10.1002/adma.201902365.

(75) Shi, J.; Chang, N.; Li, C.; Mei, J.; Deng, C.; Luo, X.; Liu, Z.; Bo, Z.; Dong, Y. Q.; Tang, B. Z. Locking the phenyl rings of tetraphenylethene step by step: understanding the mechanism of aggregation-induced emission. Chem. Commun. 2012, 48 (86), 10675-10677.

(76) Zhang, G.-F.; Chen, Z.-Q.; Aldred, M. P.; Hu, Z.; Chen, T.; Huang, Z.; Meng, X.; Zhu, M.-Q. Direct validation of the restriction of intramolecular rotation hypothesis via the synthesis of novel ortho-methyl substituted tetraphenylethenes and their application in cell imaging. Chem. Commun. 2014, 50 (81), 12058-12060.

(77) Lu, H. G.; Zheng, Y. D.; Zhao, X. W.; Wang, L. J.; Ma, S. Q.; Han, X. Q.; Xu, B.; Tian, W. J.; Gao, H. Highly Efficient Far Red/Near-Infrared Solid Fluorophores: Aggregation-Induced Emission, Intramolecular Charge Transfer, Twisted Molecular Conformation, and Bioimaging Applications. Angew. Chem., Int. Edit. 2016, 55 (1), 155-159.

(78) Huang, Y. H.; Mei, J.; Ma, X. A novel simple red emitter characterized with AIE plus intramolecular charge transfer effects and its application for thiol-containing amino acids detection. Dyes Pigment. 2019, 165, 499-507.

(79) Wang, H.; Ji, X.; Li, Y.; Li, Z.; Tang, G.; Huang, F. An ATP/ATPase responsive supramolecular fluorescent hydrogel constructed via electrostatic interactions between poly(sodium $p$-styrenesulfonate) and a tetraphenylethene derivative. J. Mater. Chem. B 2018, 6 (18), 2728-2733.

(8o) Sun, H. W.; Zhang, Y.; Yan, W.; Chen, W. X.; Lan, Q.; Liu, S. W.; Jiang, L.; Chi, Z. G.; Chen, X. D.; Xu, J. R. A novel ultrasound-sensitive mechanofluorochromic AIE-compound with remarkable blue-shifting and enhanced emission. J. Mater. Chem. C 2014, 2 (29), 5812-5817.

(81) Fateminia, S. M. A.; Wang, Z. M.; Goh, C. C.; Manghnani, P. N.; Wu, W. B.; Mao, D.; Ng, L. G.; Zhao, Z. J.; Tang, B. Z.; Liu, B. Nanocrystallization: A Unique Approach to Yield Bright Organic Nanocrystals for Biological Applications. Adv. Mater. 2017, 29 (1), 1604100.

(82) Li, Z.; Zheng, Z.; Su, S.; Yu, L.; Wang, X. L. Preparation of a High-Strength Hydrogel with Slidable and Tunable Potential Functionalization Sites. Macromolecules 2016, 49 (1), 373-386.

(83) Li, Z.; Zheng, Z.; Su, S.; Yu, L.; Wang, X. Hydroxypropyl- $\beta$-CD vs. Its $\alpha$-Homologue for a $3 D$ Modified Polyrotaxane Network Formation and Properties: the Relationship between Modified CD and Polymer Revealed through Comparison. Soft Matter 2016, 12 (34), 7089-7101. 
time $\stackrel{\text { simoultaneous fluorescence color and brightness changes and complex shape deforamtion }}{\longrightarrow}$
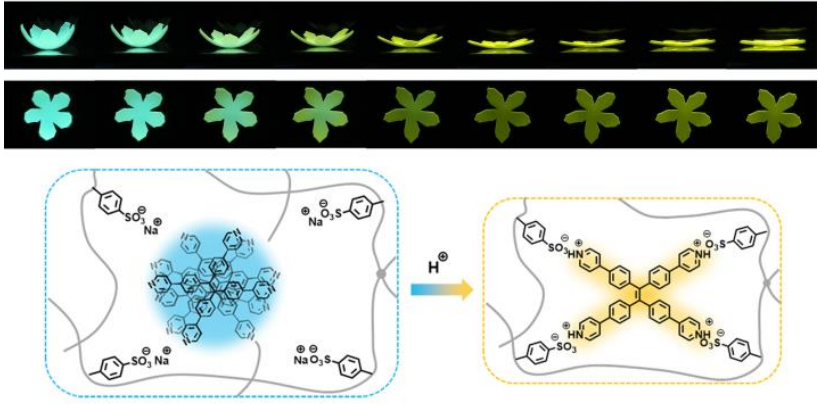